\title{
Longitudinal bone growth of the human femur
}

\author{
H. A. SisSONS \\ M.D., F.R.C.P., F.R.C.S., F.R.C.Path. \\ N. F. KEMBER* \\ Ph.D., D.Sc., F.Inst.P. \\ Royal National Orthopaedic Hospital, London, and \\ *Medical College, St Bartholomew's Hospital Medical School, London
}

\begin{abstract}
Summary
A study is presented of the histological structure and growth rate of the growth plate at the distal end of the femur in normal children. From a comparison of quantitative histological information from postmortem specimens with measurements on serial radiographs it is estimated that the distal growth plate contributes about $66 \%$ of the total longitudinal growth of the bone. The marked differences between rodent and man indicate that caution is required in extrapolating data from these animals to man.
\end{abstract}

RECENT years have seen considerable advances in our understanding of the relationship between the histological structure of the epiphyseal cartilage growth plate, the rates of division of the cartilage cells and the overall growth rates of the plates and the bones concerned. Virtually all this work has been done on small laboratory animals, and comparable human information is very scanty apart from papers by Haines and Mohiuddin (1959) and Haines (1975).

Recently, a study was made of the kinetics of longitudinal bone growth in man, the results being summarized in the present paper (Table 1). For a more detailed account of the findings, see Kember and Sissons (1976). Two types of information were necessary: (i) histological preparations of normal human epiphyseal plates at various stages of growth; (ii) the observed growth rates of those parts of

TABLE 1. Bone growth: a comparison of the distal growth plates of the femur in the rat at 30 days and in man aged 5 years

\begin{tabular}{lcc}
\hline & Rat & Man \\
\hline Plate thickness & $480 \mu \mathrm{m}$ & $500 \mu \mathrm{m}$ \\
Length of cell columns & $35 \mathrm{cells}$ & $50 \mathrm{cells}$ \\
Size of hypertrophic cells & $50 \mu \mathrm{m}$ & $30 \mu \mathrm{m}$ \\
Daily growth rate & $250 \mu \mathrm{m}$ & $35 \mu \mathrm{m}$ \\
Cells formed per day & 5 & 1 \\
Life span of cells & 7 days & 50 days \\
Intermitotic period of & 2 days & 20 days \\
proliferating cells & 6 months & 15 years \\
Period of growth & 1 cm & $20 \mathrm{~cm}$ \\
Total linear growth & & \\
\hline
\end{tabular}

individual bones where these growth plates are situated.

In the absence of material labelled with tritiated thymidine, as used in animal studies (Kember, 1972), histological preparations were used of the distal growth plate from the femora of a number of normal growing children. Serial radiographs of children's knees during the growth period, taken as part of Professor J. M. Tanner's Harpenden Growth Study at the Institute of Child Health (Tanner, Whitehouse and Takaishi, 1966) were also examined and used to measure the rate of longitudinal bone growth.

In laboratory animals, radio-opaque metab $\overrightarrow{0}$ markers or tetracycline labelling of bone provide 간. suitable reference points for measuring longitudinas bone growth. In the radiographs of children's knees Harris's transverse lines of arrested growth were used (Harris, 1933), being present in about $25 \%$ of the films examined. These lines are transverse condensations of bone trabeculae formed when the cartilage plate is in a given situation relative to the shaft of the bone; they can persist for many years and form a convenient radiographic landmark from which to measure the distance of the distal growth plate of the femur, and thus the longitudinal growth rate of this structure.

From the measurements made, the age-specific growth rates for the distal femur alone are plotted for boys (Fig. 1) and for girls (Fig. 2).

Unfortunately, for children under the age of 5 years and over 14 years data were scanty, as may be noted from these diagrams in which each point plotted represents the estimated growth rate for an individual child at that particular age. The dimorphic pattern of juvenile bone growth is well shown. Published reports of femoral growth rates almost entirely refer to the whole bone and not specifically to that portion derived from a single growth plate, as presented in Figs. 1 and 2 where the lines plotted represent mean values. In children aged 5-8 years, the mean growth velocity for the distal femur was about $1.4 \mathrm{~cm} /$ year which may be compared with American figures of $2 \cdot 2 \mathrm{~cm} /$ year for the 


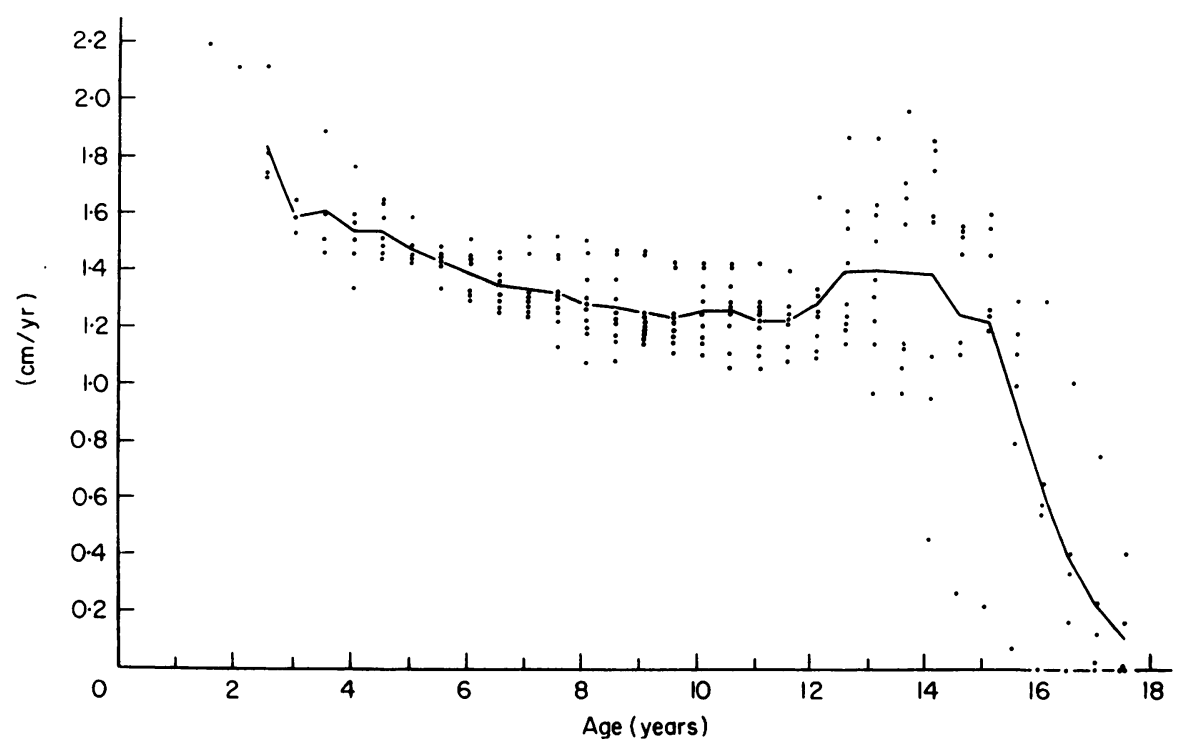

Fig. 1. Longitudinal growth of distal plate of femur, in boys, Each point represents a measured growth rate, while the continuous line joins the mean values for each age.

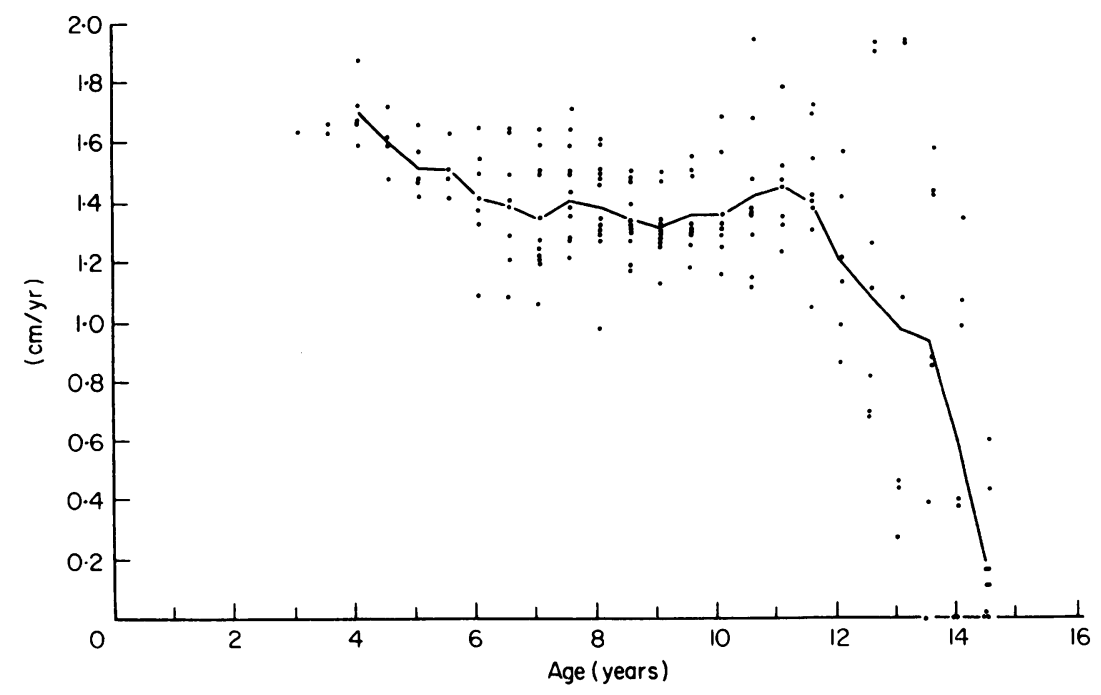

FIG. 2. Longitudinal growth of distal plate of femur, in girls.

entire femur for the same period of time. Assuming a valid comparison between normal British and American children, the distal femoral plate thus contributes about $66 \%$ of the total linear growth of the bone.

\section{Histology}

Femora for histological study were available from twelve children between birth and 14 years of age. Complete sagittal slices of the distal femur were fixed in formalin, decalcified, and conventional thin celloidin sections stained with haematoxylin and eosin.

At birth, this epiphyseal cartilage plate is not a well defined structure, but at 16 months of age, as later, a well defined band of cartilage forms its upper 


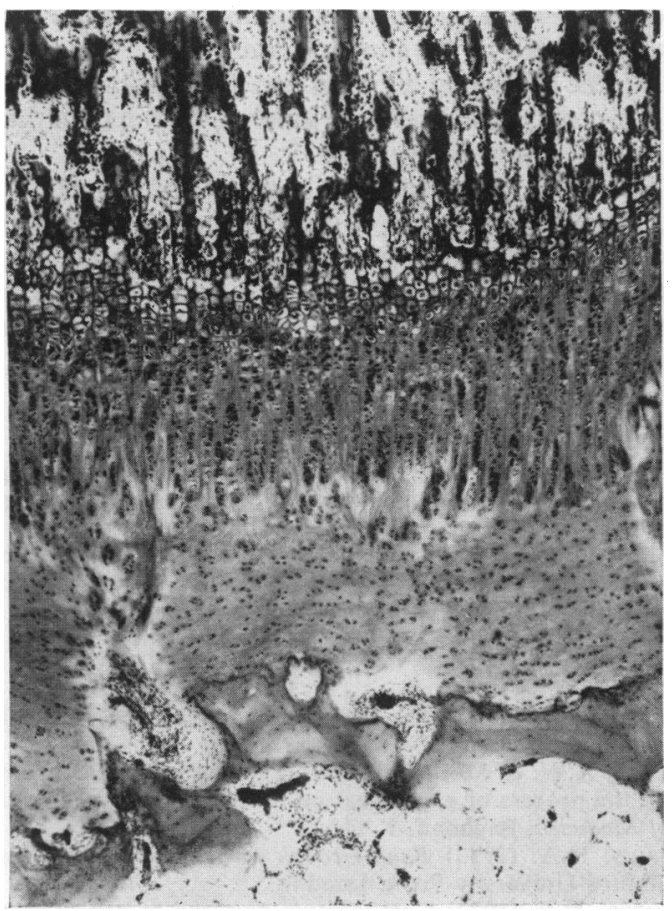

FIG. 3. Female, age 16 months. Vertical sagittal section through growth cartilage of distal femur. HE, $\times 65$.

surface (Fig. 3). This consists of two markedly different zones: (i) a columnar zone with oriented vertical rows of cartilage cells; (ii) beneath this (i.e. distally in the femur) is a zone where the cells are either separate or in small groups. This characteristic zone in man is absent in rats and rabbits, and is referred to as the inert zone of the plate. It does not seem to be a reserve area for cell proliferation as it persists from early childhood without much change until epiphyseal fusion.

At higher magnification, the cartilage cell columns also consist of two zones. Distally there is a region of proliferating cells; proximally a row of maturing and hypertrophic cells, and it is necessary to know the numbers and dimensions of these cells in order to determine the cell kinetics by comparison with the observed growth rate at a given age of this part of the bone. On the metaphyseal aspect of the hypertrophic cell zone, vascularization takes place, the intercolumnar matrix persisting as conspicuous cartilage cores in the new trabeculae.

These features of the growth plate do not change much either in appearance or in relative size between the ages of 5 and 8 years. Later, when fusion occurs, it becomes thinner (Fig. 4), its width varies and there are only small, irregular cell columns together with

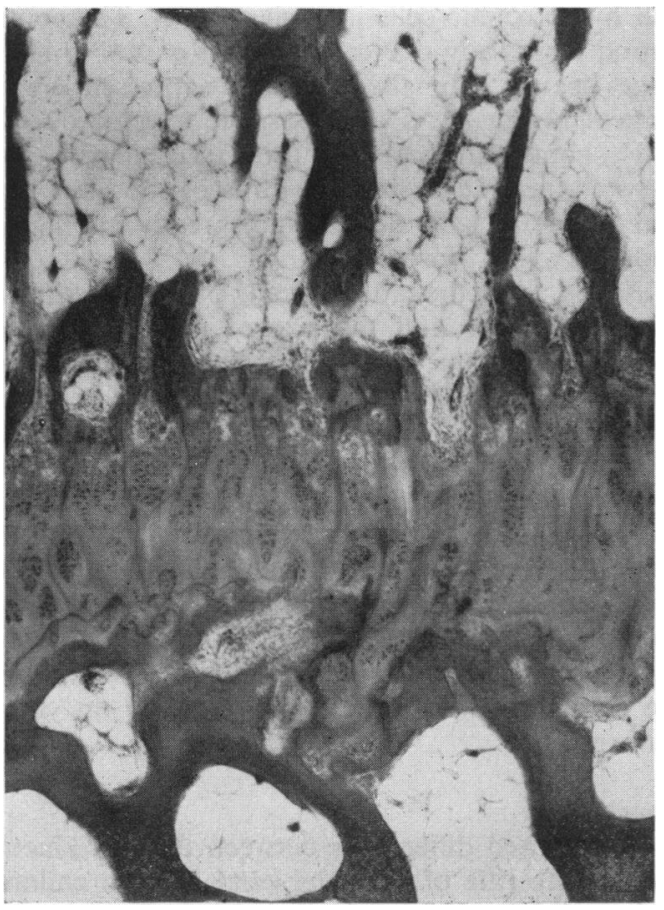

FIG. 4. Female, age 14 years. Vertical sagittal section through growth cartilage of distal femur. HE, $\times 65$.

progressive bony replacement; and eventually only small residual islets of cartilage to indicate its former site. By contrast, the rat's growth cartilage persists, but is inactive.

\section{Cell kinetics of the growth plate}

In quantitative histological studies of the cartilage cell columns certain parameters were measured: (a) the total thickness of the growth plate; (b) the thickness of the inert and columnar zones; (c) the maximum height of the proliferating, mature and hypertrophic cells in the columns. There is some difficulty in defining precisely in man the regions of these several types of cells in the columns. In experimental animals, e.g. the rabbit, this can be readily done by means of injections of tritiated thymidine which at mitosis mark selectively the dividing cells. Extrapolating from previous work in the rabbit, whose cartilage cell columns contain approximately the same number of cells as the growing child, similar proportions of cells were assumed, i.e. of the non-hypertrophic cells $50 \%$ were proliferating and $50 \%$ maturing.

Measurements were made on many growth plates and with the assumption mentioned above permitted estimates of the numbers of proliferating, maturing 
and hypertrophic cells in each column. The contribution which any one cartilage cell makes to linear bone growth can therefore be expressed by its vertical height. It is thus possible to plot out the height contribution of each form of cartilage cell to the column. The observed widths of the inert zone and columnar zone do not vary greatly in children under 10 years of age.

Finally, from the radiologically observed values of the growth rate and the size of the hypertrophic cartilage cells, one can estimate the number of new cells produced daily. From the number of cells in a column, one can estimate the time which elapses between the formation of a cartilage cell at the epiphyseal end of the column and its dissolution at the metaphyseal extremity. Moreover, knowing the growth rate of the whole system and the number of proliferating cells, one can determine the duration of their inter-mitotic period. Some mean values for children aged 5 years are shown in Table 1 where they are compared with similar observations on young growing rats.

\section{Conclusions}

The marked differences between the cell kinetics and growth rate of the epiphyseal plate in children and small laboratory animals, such as the rat, must be emphasized.

(1) In the rat, the growth rate is very much faster$250 \mu \mathrm{m} /$ day compared with $35 \mu \mathrm{m} /$ day in man.

(2) The number of cartilage cells produced daily is much greater in the rat, although they are of rather similar size to those of man.

(3) The prolonged continuation of human growth-14 to 15 years-is responsible for the much greater length of the human femur.

(4) The estimated life span and inter-mitotic period of the proliferating cells are much longer in man than in small laboratory animals.

The assumptions made above in applying rabbit data for the proportion of proliferating and maturing cartilage cells in the human growth plate columns require direct confirmation in human material by similar labelling techniques, and suitable fresh tissue from amputation specimens is urgently required.

\section{Acknowledgments}

The authors are indebted to Professor J. M. Tanner and his colleagues in the Department of Growth and Development at the Institute of Child Health for making available for this study the radiographs of children from the Harpenden Growth Study. Figs 1-4 are reproduced by courtesy of the Editor of the Journal of Bone and Joint Surgery.

\section{References}

HAINES, R.W. (1975) The histology of epiphyseal union in mammals. Journal of Anatomy, 120, 1.

Haines, R.W. \& Mohiuddin, A. (1959) A preliminary note on the process of epiphyseal union. Journal of the Faculty of Medicine. Baghdad, 1, 141.

HARris, H.A. (1933) Bone Growth in Health and Disease. Oxford University Press, London.

Kember, N.F. (1972) Comparative patterns of cell division in epiphyseal cartilage plates in the rat. Journal of Anatomy, 111, 137.

Kember, N.F. \& SisSons, H.A. (1976) Quantitative histology of the human growth plate. Journal of Bone and Joint Surgery, 58B, 426.

TANNER, J.M., Whitehouse, R.H. \& TAKaIShI, M. (1966) Standards from birth to maturity for height, weight, height velocity and weight velocity: British children. Archives of Disease in Childhood, 41, 454. 